Article

\title{
Some Theoretical Aspects of Magnetars
}

\author{
Monika Sinha \\ Indian Institute of Technology Jodhpur, Karwar 342 037, Jodhpur District, India; ms@iitj.ac.in; \\ Tel.: +91-291-2841-242
}

Received: 31 January 2018; Accepted: 4 May 2018; Published: 9 May 2018

\begin{abstract}
Magnetars have been observationally determined to have surface magnetic fields of order of $10^{14}-10^{15} \mathrm{G}$, and the implied internal field strength may be even larger. We discuss the effect of strong field on the dense matter expected to be inside neutron stars. We describe the microphysics, phenomenology, and astrophysical implications of strong field induced unpairing effect that may occur in magnetars, if the local magnetic field in the core of a magnetar exceeds a critical value. The density dependence of the pairing of proton condensate implies that the critical value required for the unpairing effect to occur is maximal at the crust-core interface and decreases towards the center of the star. As a consequence, magnetar cores with homogeneous constant fields will be partially superconducting for "medium-field" magnetars, whereas "strong-field" magnetars will be void of superconductivity. We also discuss its effect on some observational phenomena which depend on the nature and composition of matter inside neutron stars.
\end{abstract}

Keywords: neutron stars; nuclear matter; strong magnetic fields; quark matter; superconductivity; neutrino emissivity

\section{Introduction}

The densest matter in the universe is found inside neutron stars with average density of the order of $10^{14} \mathrm{~g} / \mathrm{cm}^{3}$. The matter density varies inside the neutron stars; from crust to center, it increases. Theoretical prediction of existence of neutron star was first established with the observation of pulsars-the pulsating astrophysical objects. Radiation from these objects comes in pulses with periods in the range of ms-s. These observations [1] indicate that the radiation from rotating neutron stars is directional, which is the result of the dipolar magnetic field persisting on the neutron star surface. From these observations, it was soon established that pulsars are neutron stars with strong surface magnetic fields ranging from $10^{8}$ to $10^{12-13} \mathrm{G}[2,3]$.

However, later on, some astrophysical objects, namely soft gamma repeaters (SGRs) and anomalous X-ray pulsars (AXPs), were observed [4-10]. They are believed to be neutron stars having very strong surface magnetic fields of the order of $10^{14}-10^{15} \mathrm{G}$. Since their discovery, the source of energy was somewhat a mystery. For example, SGR bursts are much more luminous than ordinary $\mathrm{X}$-ray bursts. Moreover, unlike ordinary X-ray bursts, SGR bursts exhibit absolutely no correlation between their energy and the time gap from the previous burst. This indicates that the bursts are not caused by accretion. On the other hand, the spectra of AXPs are softer than that of typical accreting $\mathrm{X}$-ray pulsars, and the spin down rate of AXPs is too low to power X-ray emission. Therefore, the radiation from AXPs is neither accretion-powered nor rotation-powered. The energy released by SGRs and AXPs and the peak luminosities of the SGR bursts can be explained if the source objects are modeled as neutron stars having surface magnetic fields $\sim 10^{14}-10^{15} \mathrm{G}$. For reviews of the properties of these objects, see [11] and references therein. Such objects are called magnetars. In 1992, Thompson and Duncan [4] showed that a strong magnetic field, as strong as $\sim 10^{16} \mathrm{G}$, can be built up inside a neutron star if the star is born with large rotational frequency. Naturally, during the last few decades, 
due to their extremely powerful magnetic fields, magnetars have attracted increasing attention in terms of range of interests: from properties of dense matter to the structure and evolution of neutron stars in strong magnetic fields.

The macroscopic properties of neutron stars as well as some internal processes, such as cooling, magnetic field evolution, and reheating, depend strongly on properties of matter in strong magnetic fields, if the central fields are sufficiently strong. For massive neutron stars, the density at the inner core may be well above $2 n_{0}, n_{0}$ being the nuclear saturation density. Many plausible theories and models for the composition of matter at this density are floating around to reproduce the observed properties of the neutron stars. These include nuclear matter, hyper-nuclear matter, strange quark matter, matter with some boson condensates, and so on. Moreover, inside the compact objects the matter may be in a normal state or in a superfluid state. Although the direct evidence of superfluid neutron star core is absent, many observed phenomena such as pulsar glitches with post glitch relaxations and the fast cooling of young neutron star Cassiopeia A, can be understood, to a great extent, in terms of the superfluidity inside the neutron star core. Here, we discuss the properties of dense matter relevant for compact stellar objects under the influence of a strong magnetic field with two models of neutron star matter: baryonic matter with and without hyperons and the strange quark matter. In addition, we discuss the superconducting nature of proton fluid inside the neutron star in the presence of a strong magnetic field.

\section{Anisotropic Nature of Matter}

In the presence of a magnetic field, the motion of charged particles is Landau-quantized. Considering the $z$ direction along the present static magnetic field $\mathbf{B}$, the energy of a particle of mass $m$ and charge $Q$ in a unit of proton charge $e$ is modified as

$$
\epsilon_{n}=\sqrt{k_{z}^{2}+m^{2}+2 n e|Q| B}
$$

where $\epsilon_{n}$ is the energy in the $n$-th Landau level, and $k_{z}$ is the component of momentum along the $z$ direction.

Now, the total energy density and the pressure of the combined system can be obtained from the energy momentum tensor of the system

$$
\begin{gathered}
T^{\mu v}=T_{m}^{\mu v}+T_{f}^{\mu \nu} \\
T_{m}^{\mu v}=E_{m} u^{\mu} u^{v}-P_{m}\left(g^{\mu v}-u^{\mu} u^{v}\right)+\frac{1}{2}\left(M^{\mu \lambda} F_{\lambda}^{v}+M^{v \lambda} F_{\lambda}^{\mu}\right)
\end{gathered}
$$

is the matter part and

$$
T_{f}^{\mu \nu}=-\frac{1}{4 \pi} F^{\mu \lambda} F_{\lambda}^{v}+\frac{1}{16 \pi} g^{\mu \nu} F^{\rho \sigma} F_{\rho \sigma}
$$

is the field part. Here, $E_{m}$ is the matter energy density, $P_{m}$ is the thermodynamic pressure, $M^{\mu v}$ is the magnetization tensor of the matter, and $F^{\mu v}$ is the electromagnetic field tensor. Now, with the presence of the magnetic field only and with the above-mentioned choice of the axes, Equations (3) and (4) reduce, respectively, to [12-15]

$$
T_{m}^{\mu \nu}=\left(\begin{array}{cccc}
E_{m} & 0 & 0 & 0 \\
0 & P_{m}-M B & 0 & 0 \\
0 & 0 & P_{m}-M B & 0 \\
0 & 0 & 0 & P_{m}
\end{array}\right)
$$


and

$$
T_{f}^{\mu \nu}=\left(\begin{array}{cccc}
\frac{B^{2}}{8 \pi} & 0 & 0 & 0 \\
0 & \frac{B^{2}}{8 \pi} & 0 & 0 \\
0 & 0 & \frac{B^{2}}{8 \pi} & 0 \\
0 & 0 & 0 & -\frac{B^{2}}{8 \pi}
\end{array}\right) .
$$

Thus, for the whole system, the total energy density is

$$
E=E_{m}+\frac{B^{2}}{8 \pi}
$$

and the pressure becomes anisotropic with the pressure in the plane perpendicular to the field

$$
P_{\perp}=P_{m}-M B+\frac{B^{2}}{8 \pi}
$$

and in the parallel direction to the field

$$
P_{\|}=P_{m}-\frac{B^{2}}{8 \pi} .
$$

Thus, the presence of magnetic field leads to anisotropicity in pressure.

\subsection{Baryonic Matter}

The simplest model of matter inside neutron stars is nucleonic matter composed of nucleons with some electrons as leptons. Within this model, there is a possibility of appearance of heavier baryons and leptons with the increase in density towards the center of the star. Thus, most general description of matter is baryonic matter composed of nucleons as well as hyperons-known as the hyper-nuclear matter.

Among many ways dense baryonic matter can be described by the non-linear Walecka model, which is based on a relativistic mean field approach [16-21] and can successfully describe both the elastic scattering and nuclear saturation properties. Within this model, the baryons interact among themselves through mesons. Considering three meson fields of the isoscalar-scalar meson $\sigma$, the isoscalar-vector meson $\omega$, and the isovector-vector meson $\rho$, within the non-linear Walecka model, the energy density of matter in the presence of the magnetic field is [15]

$$
E_{m}=\sum_{b} E_{b}+\sum_{b^{\prime}} E_{b^{\prime}}+\sum_{l} E_{l}+\frac{1}{2} m_{\sigma}^{2} \sigma^{2}+U(\sigma)+\frac{1}{2} m_{\omega}^{2} \omega^{0^{2}}+\frac{1}{2} m_{\rho}^{2} \rho_{3}^{0^{2}}
$$

where the indices $b$ and $b^{\prime}$ indicate the uncharged and charged baryons, respectively, $l$ denotes the leptons present in the matter, and

$$
\begin{gathered}
E_{b}=\frac{1}{8 \pi^{2}} \sum_{b}\left[2 k_{F}^{(b)} \mu^{(b)^{3}}-m_{b}^{*^{2}} k_{F}^{(b)} \mu^{(b)}-m_{b}^{*^{4}} \ln \left(\frac{k_{F}^{(b)}+\mu^{(b)}}{\bar{m}_{b}}\right)\right] . \\
E_{b^{\prime}}=\frac{e|Q| B}{2 \pi^{2}} \sum_{n=0}^{n_{\max }}\left(2-\delta_{n, 0}\right)\left[k_{F, n}^{\left(b^{\prime}\right)} \mu^{\left(b^{\prime}\right)}+\left(m_{b^{\prime}}^{*^{2}}+2 n e|Q| B\right) \ln \left(\frac{k_{F, n}^{\left(b^{\prime}\right)}+\mu^{b^{\prime}}}{\sqrt{m_{b^{\prime}}^{*^{2}}+2 n e|Q| B}}\right)\right]
\end{gathered}
$$

and 


$$
E_{l}=\frac{e|Q| B}{(2 \pi)^{2}} \sum_{n=0}^{n_{\max }}\left(2-\delta_{n, 0}\right)\left[k_{F, n}^{(l)} \mu^{(l)}+\left(m_{l}^{2}+2 n e|Q| B\right) \ln \left(\frac{k_{F, n}^{(l)}+\mu^{(l)}}{\sqrt{m_{l}^{2}+2 n e|Q| B}}\right)\right] .
$$

In Equations (11)-(13), $k_{F}=\sqrt{\mu^{2}-m^{*^{2}}}$ and $k_{F, n}=\sqrt{\mu^{2}-m^{*^{2}}-2 n e|Q| B}, \mu$ being the Fermi energy of the respective particle and $m^{*}$ the effective mass of baryons. The matter pressure is derived from Equation (10) using the thermodynamic relation

$$
P_{m}=\sum_{b} \mu_{b} n_{b}+\sum_{l} \mu_{l} n_{l}-E_{m}
$$

For detailed calculations, see [15].

Here, we illustrate the result numerically with the choice of potential parameters as the binding energy $E / B=-16.3 \mathrm{MeV}$, the saturation density $n_{0}=0.153 \mathrm{fm}^{-3}$, the asymmetry energy coefficient $a_{a s y}=32.5 \mathrm{MeV}$, the incompressibility $K=240 \mathrm{MeV}$, and the effective nucleon mass at the saturation $m^{*} / m=0.8$ compatible with bulk properties of nuclear matter $[15,18-21]$. The potential parameters in the hyperonic sector are fixed to reproduce the potential depth of the hyperons obtained from the experimental data of respective hypernulei as $U_{\Lambda}=-30 \mathrm{MeV}[22,23], U_{\Xi}=-18 \mathrm{MeV}$ [23], and $U_{\Sigma}=30 \mathrm{MeV}[24]$.

The effect of the magnetic field on the EoS and abundances are important when the field strength is high enough. Figure 1 shows the dependence of pressure on the baryon number density in the presence of the magnetic field. Here, we consider that the field inside the star increases gradually from the surface to the center, and the variation of field strength is modeled with the density profile of the field as [25]

$$
B\left(\frac{n_{b}}{n_{0}}\right)=B_{s}+B_{c}\left\{1-\exp \left[-\beta\left(\frac{n_{b}}{n_{0}}\right)^{\gamma}\right]\right\} .
$$

The parameters $\beta$ and $\gamma$ control the variation in the magnetic field from its surface value $B_{s}$ to its central value $B_{c}$.

From the figure, it is evident that in the presence of a magnetic field, the pressure splits into two components, parallel and perpendicular to the field direction. With the varying field strength, which increases towards the center, both the parallel and perpendicular pressures are practically equal to the pressure as in the absence of a magnetic field in a lower density regime, i.e., near the surface. With the increase in density, the deviation increases. In the transverse component, the field contribution is positive and pushes it up, while in the parallel component the field contribution pulls it down due to its negative contribution. For each value of $B_{c}$, it is possible to find a set of $\beta$ and $\gamma$, for which we obtain a plateau in the pressure-density curve. This shows an onset of instability in the matter. This is due to the pressure in the parallel direction of the field, which decreases with the increase in field strength. In Figure 1, the variation in pressure for a constant magnetic field through the star is shown by dashed-dotted curves for theoretical consideration only. Within this range of field strength, the magnetization of matter is so small that any contribution from this term is negligible. Thus, the presence of a magnetic field renders matter properties anisotropic [15].

It should be noted here that the field profile taken as in Equation (15) is merely a parameterization to illustrate it varies inside the star. It neither shows nor signifies any physical phenomena-for example, that the magnetic field should be density-dependent. The internal field cannot be several orders of magnitude stronger than the surface field. In this parameterization, by tuning the parameters used in the profile, the relative strengths of the surface and center fields can be controlled. Therefore, a general picture of academic interest has been shown to be a result that may not be relevant for physical systems currently observed. 


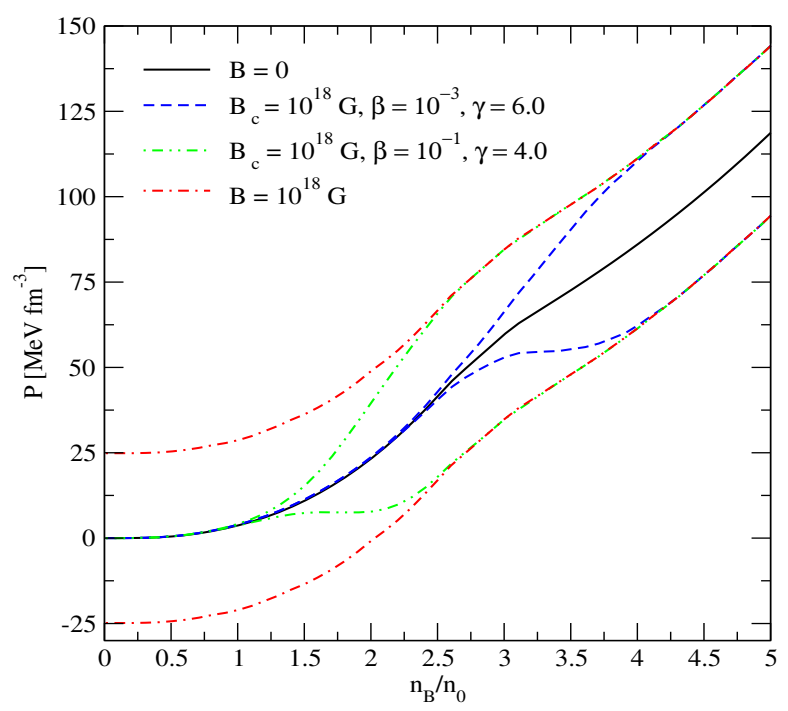

Figure 1. Dependence of total pressure on the normalized baryon number density for central magnetic fields $B_{c}=10^{18} \mathrm{G}$ and $B_{s}=10^{16} \mathrm{G}$ with different field profiles, $\beta=10^{-3}, \gamma=6$ (dashed lines), $\beta=10^{-1}$, and $\gamma=4$ (dashed-double-dotted lines). The dashed-dotted curves indicate the variation in pressure for a constant field of $B=10^{18} \mathrm{G}$ and the solid line for the field-free case. [15]. For each pair of curves, the upper branch is for $P_{\perp}$ and the lower branch for $P_{\|}$.

\subsection{Strange Quark Matter}

Another possible form of matter at high density is strange quark matter (SQM) — composed of deconfined up, down, and strange quarks. Among many proposed phenomenological models for SQM in the following discussion, we employ the model that uses the Richardson potential [26]:

$$
V\left(q^{2}\right)=-\frac{4}{9} \frac{\pi}{\ln \left[1+\left(q^{2}+m_{g}^{2}\right) / \Lambda^{2}\right]} \frac{1}{\left(q^{2}+m_{g}^{2}\right)}
$$

and density dependent quark masses as

$$
m_{i}=M_{i}+M_{q} \operatorname{sech}\left(v \frac{n_{b}}{n_{0}}\right), \quad i=u, d, s .
$$

In the potential (Equation (16)), $\Lambda$ is a scale parameter, and $m_{g}$ is the finite gluon mass given by

$$
m_{g}^{2}=D^{-2}=\frac{2 \alpha_{0}}{\pi} \sum_{i=u, d, s} k_{F}^{i} \mu_{i}^{*},
$$

which is responsible for screening in medium, with $D$ being the screening length. In this expression, $\alpha_{0}$ is the perturbative quark gluon coupling, $\mu_{i}^{*}$ the Fermi energy, and $k_{F}^{i}$ the Fermi momentum. In Equation (17), $n_{b}$ is the baryon number density, and $n_{0}$ the nuclear matter saturation density with $v$ as a parameter. With the increase in density, $n_{b}$ the quark mass $m_{i}$ falls off from its constituent value $M_{q}$ to its current value $M_{i}$. This model was originally proposed by Dey et al. [27].

In the presence of a magnetic field, the kinetic energy density of matter is modified because of Landau quantization as [28]

$$
E_{k i n}=\frac{3}{(2 \pi)^{3}} e|Q| B \sum_{n=0}^{\infty}\left(2-\delta_{n, 0}\right) \int_{0}^{2 \pi} d \phi \int_{-\infty}^{\infty} f(\epsilon) \epsilon d k_{z}
$$

where $\epsilon$ is the single particle energy as given by Equation (1), and $f(\epsilon)$ is the Fermi distribution function. The potential part is modified as [28] 


$$
\begin{array}{r}
E_{\text {pot }}^{i j}=-\frac{e^{2}\left|Q_{i}\right|\left|Q_{j}\right|}{(2 \pi)^{5}} B^{2} \sum_{n_{i}} \sum_{n_{j}}\left(2-\delta_{n_{i}, 0}\right)\left(2-\delta_{n_{j}, 0}\right) \\
\int_{0}^{2 \pi} d \phi_{i} \int_{0}^{2 \pi} d \phi_{j} \int_{-\infty}^{\infty} d k_{z}^{i} \int_{-\infty}^{\infty} d k_{z}^{j} f\left(\epsilon_{i}\right) f\left(\epsilon_{j}\right) N V\left(q^{2}\right) S
\end{array}
$$

where

$$
\begin{aligned}
N & =\frac{\left(\epsilon_{i}+m_{i}\right)\left(\epsilon_{j}+m_{j}\right)}{4 \epsilon_{i} \epsilon_{j}} \\
S & =1+\frac{k_{i}^{2} k_{j}^{2}}{\left(\epsilon_{i}+m_{i}\right)^{2}\left(\epsilon_{j}+m_{j}\right)^{2}}+\frac{2 \mathbf{k}_{i} \cdot \mathbf{k}_{j}}{\left(\epsilon_{i}+m_{i}\right)\left(\epsilon_{j}+m_{j}\right)} .
\end{aligned}
$$

The total energy density of the matter is then

$$
E_{m}=\sum_{i} E_{k i n}+\frac{1}{2} \sum_{i, j} E_{\text {pot }}^{i j} \quad i, j=u, d, s
$$

and the thermodynamic pressure is

$$
p=\sum_{i} \mu_{i} n_{i}+T s-E, \quad i=u, d, s
$$

where

$$
s=-\frac{3}{(2 \pi)^{3}} e \sum_{i}\left|Q_{i}\right| B \sum_{n=0}^{\infty} \int_{0}^{2 \pi} d \phi \int_{\infty}^{\infty} d k_{z} \times\left\{f\left(\epsilon_{i}\right) \ln f\left(\epsilon_{i}\right)+\left[1-f\left(\epsilon_{i}\right)\right] \ln \left[1-f\left(\epsilon_{i}\right)\right]\right\}
$$

is the net entropy density. Here, we should note that the lepton abundances are negligible for SQM.

We present the effect of magnetic field on SQM within the frame work of the Richardson potential as well as the MIT bag model in Figure 2, choosing the numerical values of the parameters as $\Lambda=100 \mathrm{MeV}, v=0.333, \alpha_{0}=0.2, M_{q}=310 \mathrm{MeV}, M_{u}=4 \mathrm{MeV}, M_{d}=7 \mathrm{MeV}$, and $M=150 \mathrm{MeV}$ [28].

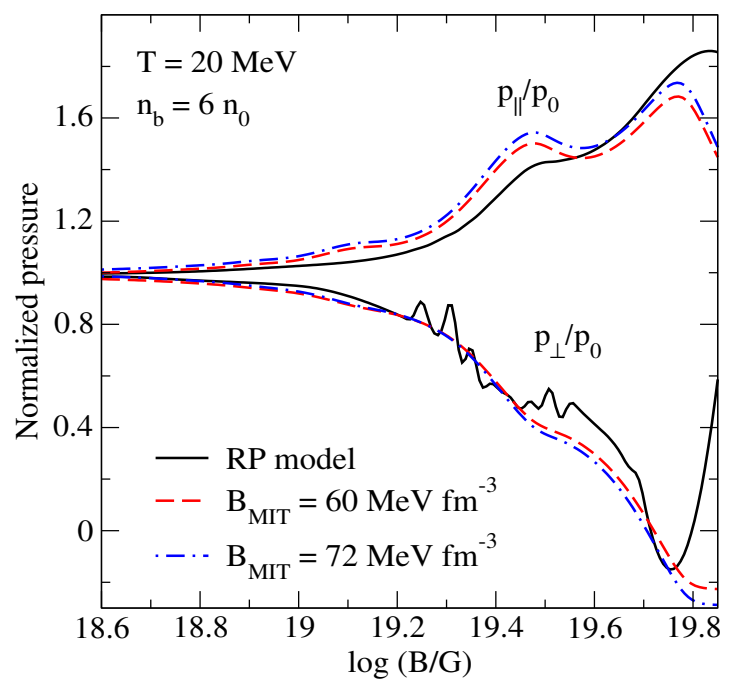

Figure 2. Variation in the normalized pressure in parallel and perpendicular directions to the magnetic field with the strength of the magnetic field at $n_{b}=6 n_{0}$ and $T=20 \mathrm{MeV}$ [28]. The solid curve is for the model under consideration, the dashed and the dash-dotted curves for the MIT bag model with values of bag constants 60 and $72 \mathrm{MeV} \mathrm{fm}^{-3}$, respectively. The upper curves correspond to the parallel pressure, the lower curves to the perpendicular pressure. 
From the figure, it is evident that, when the field is below $B=3 \times 10^{18} \mathrm{G}$, both $p_{\|}$and $p_{\perp}$ differ very little from the matter pressure in the absence of a magnetic field. Thus, the effect of the magnetic field for the SQM is not significant below $B \sim 10^{18} \mathrm{G}$. With the increase in the field, $p_{\|}$increases, whereas $p_{\perp}$ decreases.

\section{Quenched Superconductivity}

Matter inside neutron stars may exist in a superfluid state through the pair forming of the constituent particles. To study the effect of the magnetic field on matter in a superfluid state, we consider the simple nucleonic matter with electrons and muons. From a microscopic point of view with the model under consideration, protons form type-II superconductivity inside a neutron star core, although there may be some arguments for the existence of type-I proton superconductivity inside neutron stars with some recent observational data [29-34].

The variation in matter pressure and the abundances of the constituent particles with the normalized baryon density are shown in the two panels of Figure 3. For this calculation, we choose the matter EoS using density-dependent couplings as derived in [35]. The parameterization is chosen to reproduce nuclear saturation density $n_{0}=0.152 \mathrm{fm}^{-3}$, binding energy per nucleon $E / A=-16.14 \mathrm{MeV}$, incompressibility $K_{0}=250.90 \mathrm{MeV}$, symmetry energy $J=32.30 \mathrm{MeV}$, symmetry energy slope $L=51.24 \mathrm{MeV}$, and symmetry incompressibility $K_{\text {sym }}=-87.19 \mathrm{MeV}$ [36]. As mentioned earlier, the EoS as well as the composition of matter is affected by the presence of the magnetic field. However, the magnetic field effect is evident only when the interaction energy with the magnetic field for the particles are comparable with their Fermi energy. Below, the field value $\sim 10^{18} \mathrm{G}$, the effect of magnetic field on matter composition and EoS is insignificant.

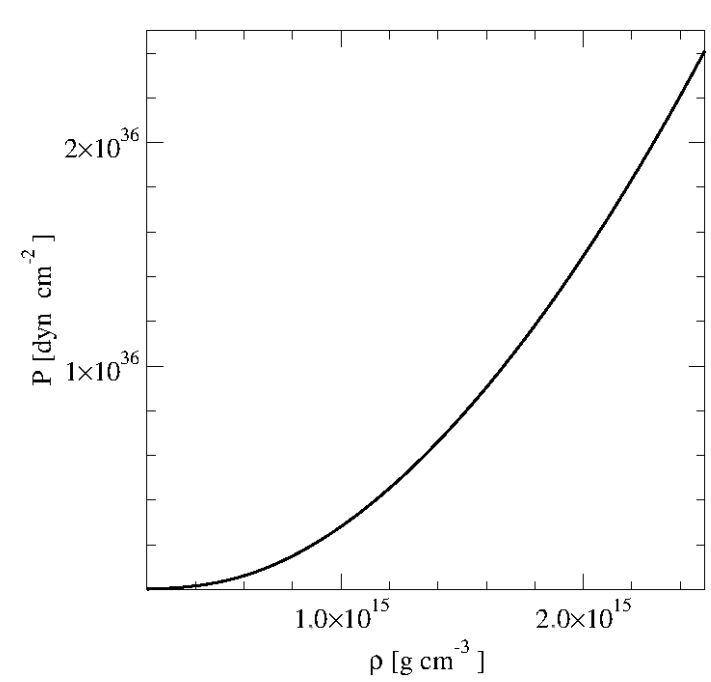

(a)

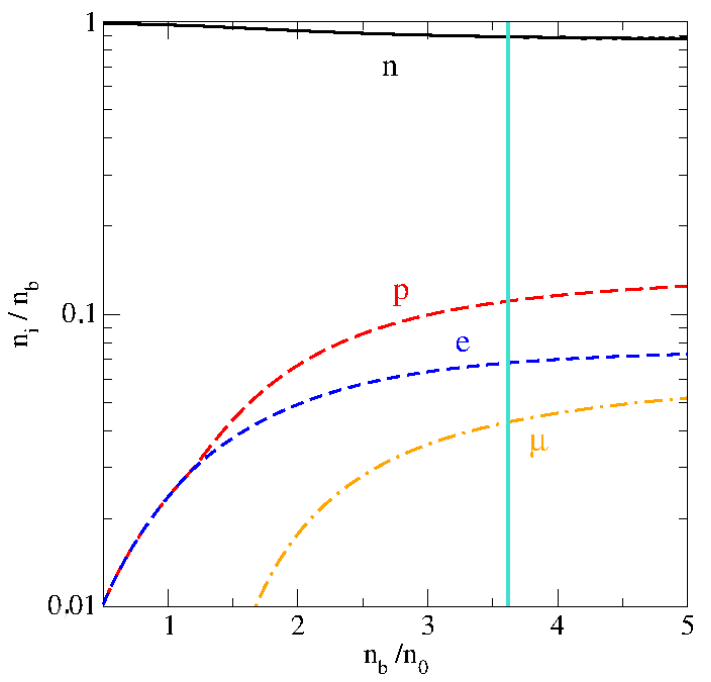

(b)

Figure 3. (a) Zero-temperature equation of state. (b) Variation in particle abundances on the normalized baryon density. The vertical line indicates the approximate Urca threshold.

In the crust of a compact star, low density neutrons pair in the ${ }^{1} S_{0}$ channel. Towards the center, as the density increases, the $S$-wave interaction becomes repulsive and the neutron pairing in the ${ }^{3} P_{2}-{ }^{3} F_{2}$ channel becomes important. However, protons are much less abundant throughout the core and hence proton pairing in the ${ }^{1} S_{0}$ channel is admissible inside the core. Figure 4 shows variation in gap energies for the dominant channels with Fermi momenta of the respective particles relevant for the interior of the neutron star adopted from [37-39]. 


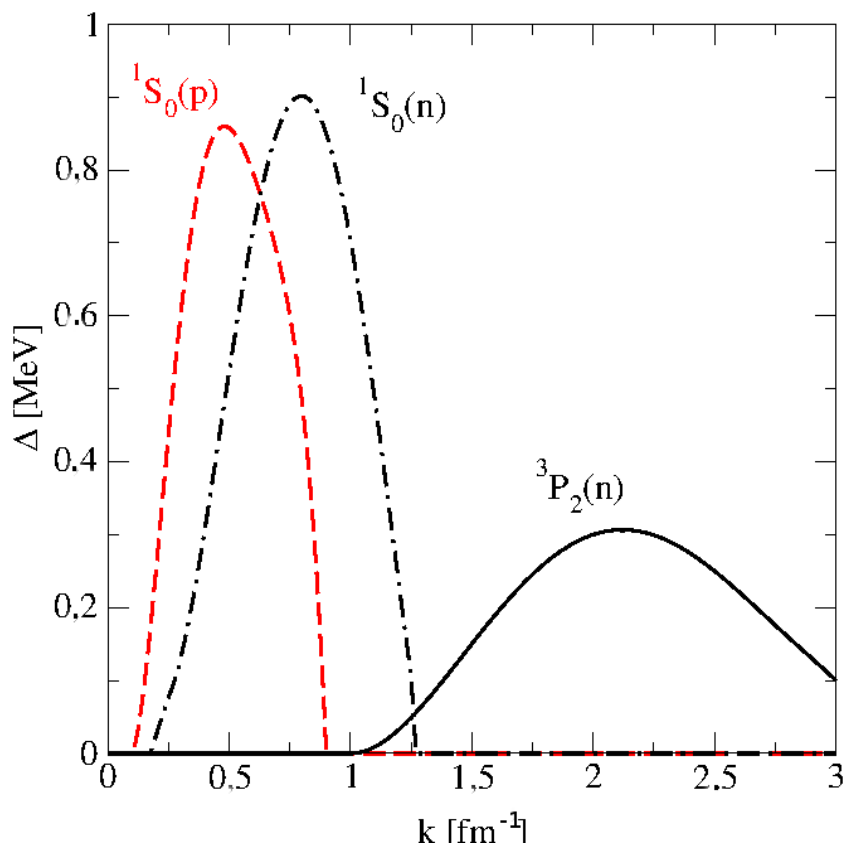

Figure 4. Variation in pairing gaps of neutrons (dash-dotted and solid lines) and protons (dashed line) on their respective Fermi-momenta.

Type-II proton superconductivity can exist if the persisting magnetic field is greater than the lower critical field $H_{c 1}$ but less than the upper critical field $H_{c 2}$. The critical field is determined by the microscopic properties of matter and can be described in terms of Ginzburg-Landau (GL) theory. The GL functional for the fluid containing both the superfluid neutron and superconducting proton can be written as [40]

$$
F[\phi, \psi]=F_{n}[\phi]+\alpha \tau|\psi|^{2}+\frac{b}{2}|\psi|^{4}+\frac{b^{\prime}}{2}|\psi|^{2}|\phi|^{2}+\frac{1}{4 m_{p}}\left|\left(-i \hbar \nabla-\frac{2 e}{c} \mathbf{A}\right) \psi\right|^{2}+\frac{B^{2}}{8 \pi}
$$

where $\psi$ and $\phi$ are the proton and neutron condensate wave-functions, $F_{n}[\phi]$ is the GL functional for neutron superfluid, $m_{p}$ is the proton mass, and $\tau=\left(T-T_{c p}\right) / T_{c p}, T_{c p}$ being the critical temperature of superconducting phase transition of protons. Here, $\alpha, b$, and $b^{\prime}$ are the constants. For detailed discussion, see [34]. Then, the upper critical field can be calculated to the linear order of $\psi$ as [40]

$$
H_{c 2}=\frac{\Phi_{0}}{2 \pi \xi_{p}^{2}}\left[1+\frac{\left|b^{\prime}\right||\phi|^{2}}{\alpha|\tau|}\right]
$$

with $\Phi_{0}$ being the flux quantum and $\xi$ the coherence length. Equation (25) reduces to the standard result [41] if $b^{\prime}=0$.

The dependence of pairing gaps on baryon density is shown in the upper panel of Figure 5, and the dependence of $H_{c 2}$ field on density is shown in the lower panel of the same figure.

The critical field has a maximum near baryon density $n_{b}=0.7 n_{0}$, which is close to the crust-core interface with $\max H_{c 2} \simeq 6.25 \times 10^{16} \mathrm{G}$. Thus, for a magnetar with interior field less than the maximum critical field will be partially superconducting. The regions where fields are less than the local critical field will be superconducting, whereas the regions where fields are greater than the local critical field are not. For this kind of magnetar where the interior field is less than $\max H_{c 2}$ the proton superconductivity will be removed from the inner core, whereas the outer core may contain the superconducting protons as clear from Figure 5. 


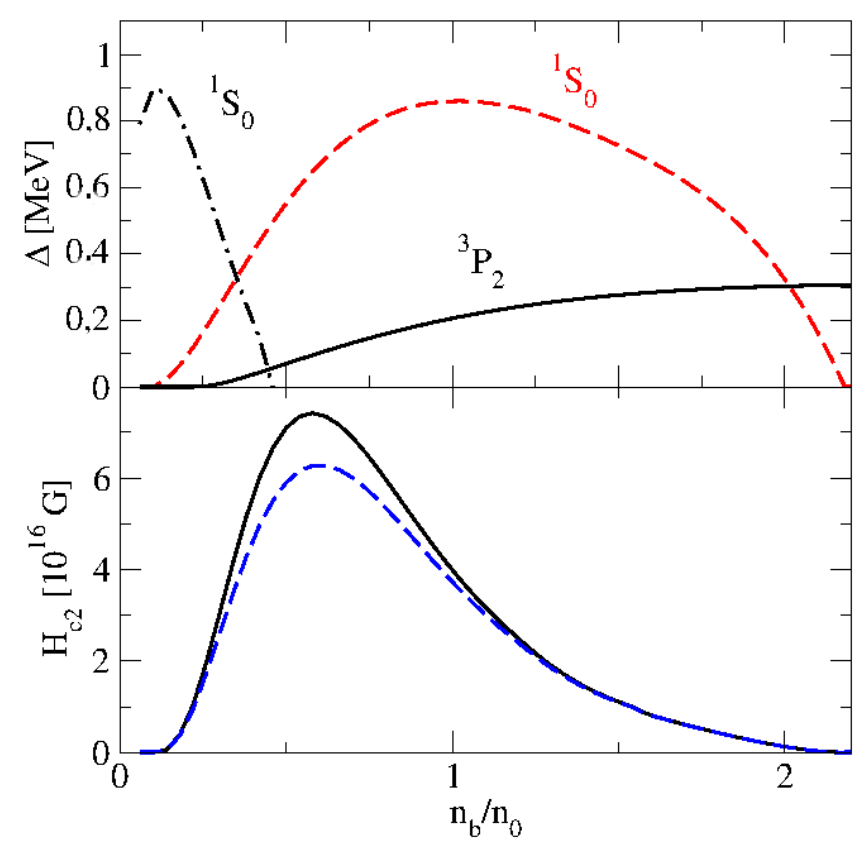

Figure 5. (Upper panel): Variation in pairing gaps for neutrons and for protons on normalized baryon density. (Lower panel): Dependence of the upper critical field on normalized baryon density with the coupling between the neutron and proton condensates (full line) and without (dashed line).

\section{Neutrino Emissivity}

The absence of superconducting protons inside the entire part of neutron star interior, or in some part of the neutron star interior in the presence of the magnetic field has great impacts on neutrino emission processes from matter inside magnetars. Here, we discuss two dominant neutrino emission processes-the direct Urca and the pair-breaking processes.

\subsection{The Direct Urca Process}

Inside the dense neutron star matter, the direct Urca process is allowed only when the proton fraction in the matter is above a threshold $Y_{\text {Urca }}=n_{p} / n_{b}>11 \%$ [42-44]. However, the presence of a strong magnetic field changes the phase space of nucleons. As a consequence, it smears out the sharp boundary between the closed and open direct Urca regimes leading the direct Urca process to be allowed even below $Y_{\text {Urca }}$ with substantial suppression in its emissivity [45-47]. The allowed and forbidden region for direct Urca in the field-free case can be described conveniently by introducing the parameter [47]

$$
x=\frac{k_{F n}^{2}-\left(k_{F e}+k_{F p}\right)^{2}}{k_{F n}^{2}} n_{F p}^{2 / 3}
$$

where $n_{F p}$ is the number of Landau levels populated by protons. The direct Urca process is forbidden for $x>0$ in the field-free case, while it can become operative in the presence of strong magnetic fields. The variation in neutrino emissivity due to the Urca process with the magnetic field in the forbidden regime is shown in Figure 6 by the dotted curve [45-47], when the matter is normal.

If matter is in a superfluid state, the available phase space for the process is restricted by proton and neutron pairing. Consequently, the direct Urca process is suppressed by an exponential factor $\exp (-\Delta / T)$ for each participating nucleon, with $\Delta$ being the relevant pairing gap and $T$ the temperature. Detailed calculations are given in $[48,49]$. The suppression in emissivity due to the presence of pairs is shown by the dashed-dotted curves in Figure 6 for two different temperatures. 


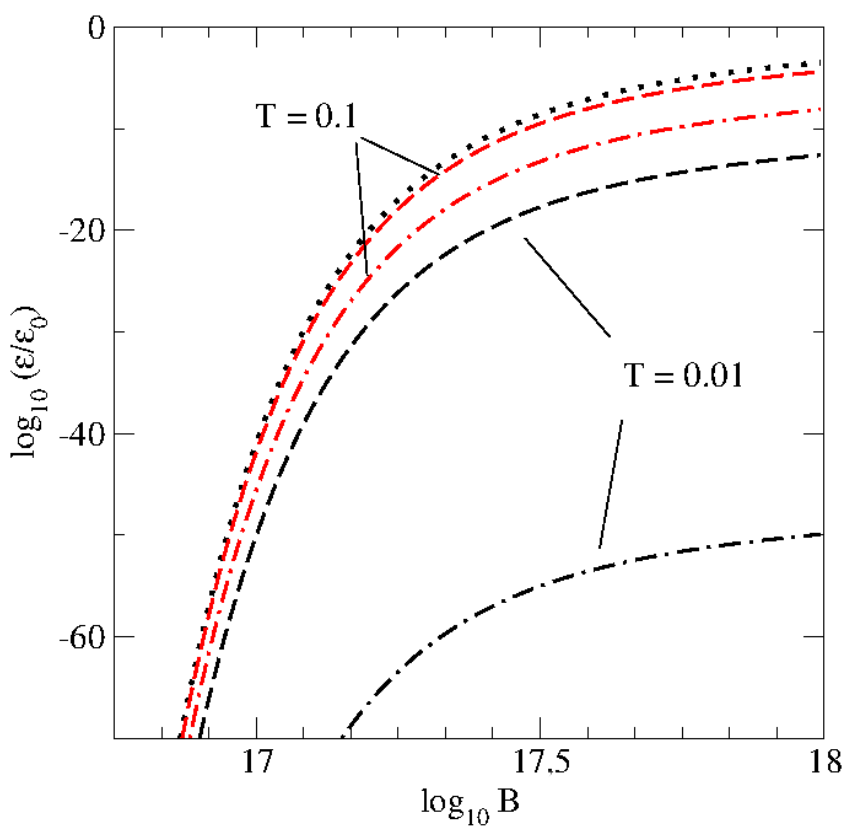

Figure 6. The direct Urca emissivity in the forbidden region in units of the zero-field emissivity at a fixed density $n=n_{0}$ and for two temperatures $T=0.01$ and $0.1 \mathrm{MeV}$. The emissivity for normal matter (dotted line) [45-47], paired neutrons and normal protons (dashed lines), and paired neutrons and protons (dashed-dotted lines) [40].

However, the presence of a strong magnetic field unpairs the proton superconductor if the field strength is larger than the local upper critical field [40]. In that case, the suppression of the Urca process due to the proton pairing gap is absent and the suppression for the pairing gap is only due to neutron pairing. This effect is shown by the dashed curves in the same figure (Figure 6). The suppression strongly deviates from that expected in the case of superconducting protons as the gap for neutron pairing in the $P$-wave channel is smaller than the one in the $S$ channel for protons (see Figure 5).

In the allowed region, the effect of magnetic field is the introduction of de-Haas-van Alfven type oscillations in the emissivity around its value in the zero $B$-field limit, as expected [45-47]. This is shown in the panel a of Figure 7, which is the case for normal matter. In the case of paired nucleons the emissivity is suppressed due to pairing gaps of both protons and neutrons in the low field regime, as shown by panel c of Figure 7. When the field crosses the value of the local upper critical field, the protons are unpaired and the suppression due to proton pairing disappears leading to enhancement in emissivity, as shown by the panel $b$ of the same Figure 7. Here it should be noted that a sharp jump seen in the emissivity is in fact a smooth transition because, in the range of fields $H_{c 1}<B<H_{c 2}$, the superconductor is in the mixed (flux-tube) state. The emissivity in the mixed state has not been studied by us, so we present only the asymptotic results for $B \leq H_{c 1}$ and $B \geq H_{c 2}$. 


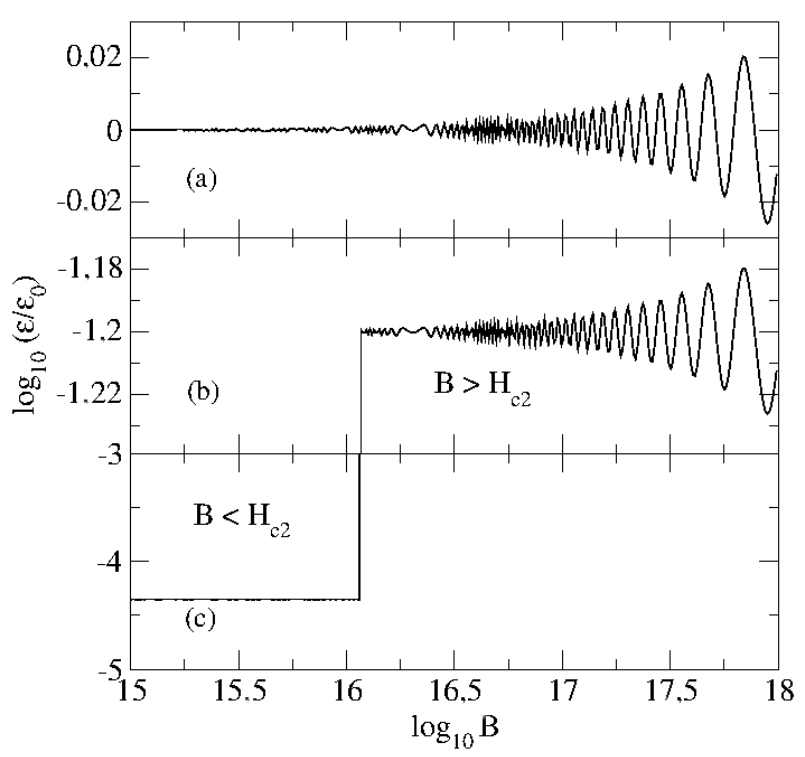

Figure 7. The direct Urca emissivity in the allowed region in units of the zero-field emissivity at fixed density $n=1.5 n_{0}$ for temperature $T=0.1 \mathrm{MeV}$. The emissivity for (a) unpaired matter, (b) paired neutrons and unpaired protons when $B>H_{c 2}$, and (c) both paired neutrons and protons when $B<H_{c 2}$.

\subsection{Pair-Breaking Processes}

The neutrino emission due to pair-breaking $(\mathrm{PB})$ from each nucleonic BCS condensates [50-56] is also affected by the unpairing of protons when the field strength is larger than the upper critical field $H_{c 2}$.

The neutrino emissivity due to pair breaking process is given by $(\hbar=c=1)$

$$
\begin{gathered}
\varepsilon_{n}=\frac{4 G_{F}^{2} m_{n}^{*} k_{F n}}{15 \pi^{5}} T^{7} a_{n}^{S / P}\left(\frac{\Delta_{n}^{S / P}}{T}\right)^{2} \mathcal{I} \\
\varepsilon_{p}=\frac{4 G_{F}^{2} m_{p}^{*} k_{F p}}{15 \pi^{5}} T^{7} a_{p}^{S}\left(\frac{\Delta_{p}}{T}\right)^{2} \mathcal{I}
\end{gathered}
$$

where $G_{F}$ is the Fermi coupling constant, the subscripts $n$ and $p$ stand for neutrons and protons, respectively, and superscripts $S$ and $P$ stand for the ${ }^{1} S_{0}$ and ${ }^{3} P_{2}$ pairing of neutrons. $\Delta_{n}^{P}$ in Equation (27) refers to the angle averaged value of the spin-triplet neutron gap, in which case it can be factored out of the integral $\mathcal{I}$. The coefficients $a^{\prime}$ s are defined as follows:

$$
\begin{gathered}
a_{n}\left({ }^{1} S_{0}\right)=\frac{4}{81} c_{n V}^{2} v_{F n}^{4}+\frac{11}{42} c_{n A}^{2} v_{F n}^{2} \chi_{n} \\
a_{p}\left({ }^{1} S_{0}\right)=\frac{4}{81} c_{p V}^{2} v_{F p}^{4}+\frac{11}{42} c_{p A}^{2} v_{F p}^{2} \chi_{p} \\
a_{n}\left({ }^{3} P_{2}\right)=\frac{c_{n A}^{2}}{2}
\end{gathered}
$$

where $\chi_{n / p}=1+(42 / 11)\left(m_{n / p}^{*} / m_{n / p}\right)^{2}, c_{n V}=1, C_{n A}=g_{A}, C_{p V}=4 \sin ^{2} \theta_{W}-1$, and $C_{p A}=-g_{A}$, with $g_{A} \simeq 1.26$ and $\sin ^{2} \theta_{W}=0.23$. 
Figure 8 displays the functions

$$
Q_{n / p}(B)=\frac{m_{n / p}^{*} k_{F n / F p}}{m_{n / p}} a_{n / p}^{S / P}\left(\frac{\Delta_{n}^{S / P}}{T}\right)^{2}
$$

which are more convenient for the present analysis than the emissivities as a whole as all other common factors appearing in the emissivity Equations (27) and (28), which are constants throughout the interior of the star that are discarded.

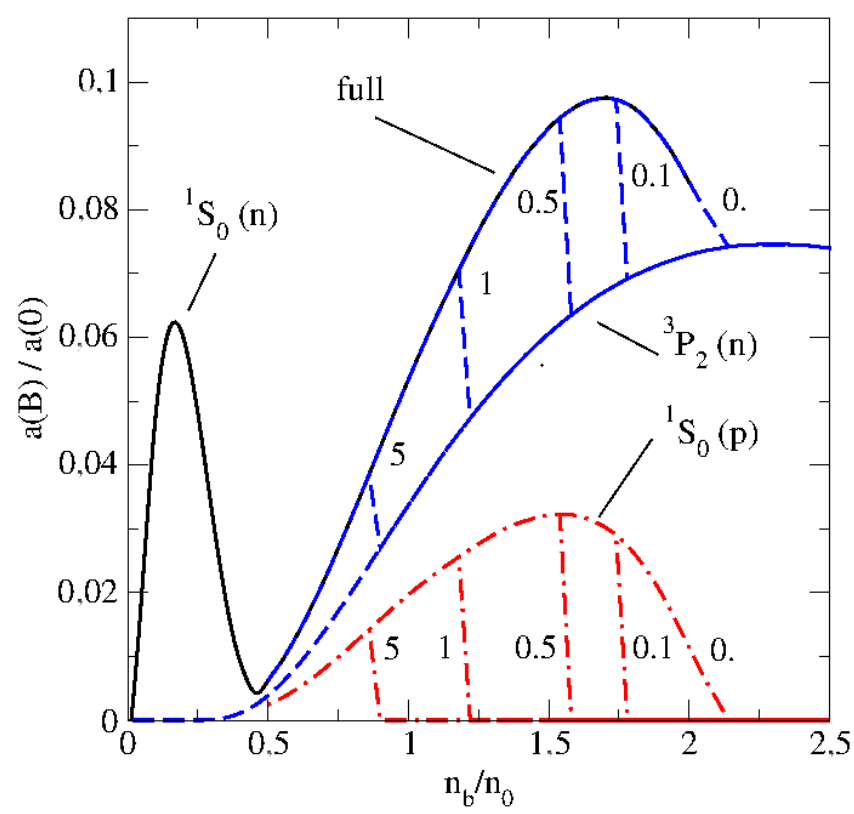

Figure 8. Neutrino emissivity via pair-breaking processes in units of zero-field emissivity as a function of normalized baryon number density for different field strengths $B_{16}=0, B_{16}=0.5, B_{16}=1$, and $B_{16}=5$. The total pair-breaking emissivity is shown by solid lines. It is a result of neutron pair emission for $n_{b} / n_{0}<0.5$, and the sum of proton and neutron pair emission for larger densities. The separate contributions of proton and neutron pairs are shown by dash-dotted and dashed lines, respectively.

In the crust of the star where $n \leq 0.5 n_{0}$, the pair-breaking emission is only due to the neutron Cooper pairs, which are unaffected by the unpairing effect. In the core where the densities are large, both the neutron and proton Cooper pair-breaking processes are present, which is shown by the solid curve in Figure 8. Assuming the constant field strength inside the star, the influence of the unpairing effect for the proton pairs is seen in the same figure by dashed-dotted curves for different constant field strengths as $B_{16}=0.1,0.5,1$, and $5 \mathrm{G}$. The presence of the field removes the proton pairs where $B>H_{c 2}$ locally. Consequently, the total emission rate reduces to its value corresponding to the rate due to only neutron pairs.

\section{Summary and Conclusions}

We have studied the properties of highly dense matter under the influence of a strong magnetic field in the context of magnetars. We have considered two different forms of matter-the baryonic matter with and without hyperons and deconfined strange quark matter. However, it should be noted that, in the present calculations, the sea effect has not been considered for simplicity, which should be considered for a more precise result. We also studied the effect of the magnetic field on the proton pair if the matter is considered in a superconducting state.

We found that the presence of a magnetic field introduces anisotropic pressure in the system and that, at a high field strength, the matter becomes unstable. The negative contribution from 
field pressure or from the interaction of matter with field to pressure leads to an instability above a critical field.

We have shown another important effect of magnetic fields on matter if the matter inside the magnetar is considered to be in a superconducting phase. This effect is the unpairing of proton condensates in the presence of a magnetic field. Consequently, magnetars are fully or partially free of proton superconductivity depending on the strength of the field inside the magnetars.

Calculating the critical field $H_{c 2}$ for the unpairing of the proton condensate, considering its coupling to the neutron condensate present, we have shown that, near the crust-core boundary, the critical field is maximum and decreases towards the center of the star. Thus, magnetars with interior fields $B<\max _{c 2}$ are partially non-superconducting, whereas magnetars with $B>\max H_{c 2}$ are fully non-superconducting, under the assumption that the field is constant throughout the interior of the star.

The unpairing of proton condensates affect neutrino emissivity. For the direct Urca, it removes the suppression of the process because the proton gap enhances the emissivity, compared to the low-field case. On the other hand, in the case of neutrino emissivity, due to the Cooper pair-breaking processes, the unpairing of protons due to a high magnetic field removes the pair-breaking emissivity for proton pairing, reducing the total pair-breaking emissivity to a low extent.

Acknowledgments: The author is grateful to Armen Sedrakian for the useful discussions. We also thank the anonymous referees for useful suggestions in modifying the text and the presentation.

Conflicts of Interest: The author declares no conflict of interest.

\section{References}

1. Hewish, A.; Bell, S.J.; Pilkington, J.D.H.; Scott, P.F.; Collins, R.A. Observation of a Rapidly Pulsating Radio Source. Nature 1968, 217, 709-713. [CrossRef]

2. Gold, T. Rotating Neutron Stars as the Origin of the Pulsating Radio Sources. Nature 1968, 218, 731-732. [CrossRef]

3. Gold, T. Rotating Neutron Stars and the Nature of Pulsars. Nature 1969, 221, 25-27. [CrossRef]

4. Duncan, R.C.; Thompson, C. Formation of very strongly magnetized neutron stars-Implications for gamma-ray bursts. Astrophys. J. 1992, 392, L9-L13. [CrossRef]

5. Usov, V.V. Millisecond pulsars with extremely strong magnetic fields as a cosmological source of gamma-ray bursts. Nature 1992, 357, 472-474.

6. Thompson, C.; Duncan, R.C. The soft gamma repeaters as very strongly magnetized neutron stars-I. Radiative mechanism for outbursts. Mon. Not. R. Astron. Soc. 1995, 275, 255-300. [CrossRef]

7. Thompson, C.; Duncan, R.C. The Soft Gamma Repeaters as Very Strongly Magnetized Neutron Stars. II. Quiescent Neutrino, X-Ray, and Alfven Wave Emission. Astro. Phys. J. 1996, 473, 322. [CrossRef]

8. Vasisht, G.; Gotthelf, E.V. The Discovery of an Anomalous X-Ray Pulsar in the Supernova Remnant Kes 73. Astrophys. J. 1997, 486, L129. [CrossRef]

9. Kouveliotou, C.; Dieters, S.; Strohmayer, T.; van Paradijs, J.; Fishman, G.J.; Meegan, C.A.; Hurley, K.; Kommers, J.; Smith, I.; Frail, D.; et al. An X-ray pulsar with a superstrong magnetic field in the soft $\gamma$-ray repeater SGR1806-20. Nature 1998, 393, 235-237. [CrossRef]

10. Woods, P.M.; Kouveliotou, C.; van Paradijs, J.; Hurley, K.; Kippen, R.M.; Finger, M.H.; Briggs, M.S.; Dieters, S.; Fishman, G.J. Discovery of a New Soft Gamma Repeater, SGR 1627-41. Astrophys. J. 1999, 519, L139-L142. [CrossRef]

11. Kaspi, V.M.; Beloborodov, A.M. Magnetars. Ann. Rev. Astron. Astrophys. 2017, 55, 261-301. [CrossRef]

12. Khalilov, V.R. Macroscopic effects in cold magnetized nucleons and electrons with anomalous magnetic moments. Phys. Rev. D 2002, 65, 056001. [CrossRef]

13. Huang, X.G.; Huang, M.; Rischke, D.H.; Sedrakian, A. Anisotropic hydrodynamics, bulk viscosities, and r-modes of strange quark stars with strong magnetic fields. Phys. Rev. D 2010, 81, 045015. [CrossRef]

14. Paulucci, L.; Ferrer, E.J.; de La Incera, V.; Horvath, J.E. Equation of state for the magnetic-color-flavor-locked phase and its implications for compact star models. Phys. Rev. D 2011, 83, 043009. [CrossRef] 
15. Sinha, M.; Mukhopadhyay, B.; Sedrakian, A. Hypernuclear matter in strong magnetic field. Nucl. Phys. A 2013, 898, 43-58. [CrossRef]

16. Walecka, J.D. A theory of highly condensed matter. Ann. Phys. 1974, 83, 491-529. [CrossRef]

17. Boguta, J.; Bodmer, A.R. Relativistic calculation of nuclear matter and the nuclear surface. Nucl. Phys. A 1977, 292, 413-428. [CrossRef]

18. Glendenning, N.K. The hyperon composition of neutron stars. Phys. Lett. B 1982, 114, 392-396. [CrossRef]

19. Glendenning, N.K. Neutron stars are giant hypernuclei? Astrophys. J. 1985, 293, 470-493. [CrossRef]

20. Glendenning, N.K. Hyperons in neutron stars. Z. Phys. Hadron. Nucl. 1987, 326, 57-64. [CrossRef]

21. Glendenning, N.K. Role of hyperons and pions in neutron stars and supernova. Z. Phys. Hadron. Nucl. 1987, 327, 295-300. [CrossRef]

22. Dover, C.; Gal, A. Sigma hypernuclei. Comments Nucl. Part. Phys. 1984, 12, 155-165.

23. Fukuda, T.; Higashi, A.; Matsuyama, Y.; Nagoshi, C.; Nakano, J.; Sekimoto, M.; Tlustý, P.; Ahn, J.K.; En'yo, H.; Funahashi, H.; et al. Cascade hypernuclei in the $\left(\mathrm{K}^{-}, \mathrm{K}^{+}\right)$reaction on ${ }^{12} \mathrm{C}$. Phys. Rev. C 1998, 58, 1306-1309. [CrossRef]

24. Bart, S.; Chrien, R.E.; Franklin, W.A.; Fukuda, T.; Hayano, R.S.; Hicks, K.; Hungerford, E.V.; Michael, R.; Miyachi, T.; Nagae, T.; et al. $\Sigma$ Hyperons in the Nucleus. Phys. Rev. Lett. 1999, 83, 5238-5241. [CrossRef]

25. Bandyopadhyay, D.; Chakrabarty, S.; Pal, S. Quantizing Magnetic Field and Quark-Hadron Phase Transition in a Neutron Star. Phys. Rev. Lett. 1997, 79, 2176-2179. [CrossRef]

26. Richardson, J.L. The heavy quark potential and the Upsilon, J/ $\psi$ systems. Phys. Lett. B 1979, 82, $272-274$. [CrossRef]

27. Dey, M.; Bombaci, I.; Dey, J.; Ray, S.; Samanta, B.C. Strange stars with realistic quark vector interaction and phenomenological density-dependent scalar potential. Phys. Lett. B 1998, 438, 123-128. [CrossRef]

28. Sinha, M.; Huang, X.G.; Sedrakian, A. Strange quark matter in strong magnetic fields within a confining model. Phys. Rev. D 2013, 88, 025008. [CrossRef]

29. Sedrakian, D.M.; Sedrakian, A.D.; Zharkov, G.F. Type I superconductivity of protons in neutron stars. Mon. Not. R. Astron. Soc. 1997, 290, 203-207. [CrossRef]

30. Link, B. Constraining Hadronic Superfluidity with Neutron Star Precession. Phys. Rev. Lett. 2003, 91, 101101. [CrossRef] [PubMed]

31. Buckley, K.B.; Metlitski, M.A.; Zhitnitsky, A.R. Neutron Stars as Type-I Superconductors. Phys. Rev. Lett. 2004, 92, 151102. [CrossRef] [PubMed]

32. Buckley, K.B.; Metlitski, M.A.; Zhitnitsky, A.R. Vortices and type-I superconductivity in neutron stars. Phys. Rev. C 2004, 69, 055803. [CrossRef]

33. Sedrakian, A. Type-I superconductivity and neutron star precession. Phys. Rev. D 2005, 71, 083003. [CrossRef]

34. Alford, M.; Good, G.; Reddy, S. Isospin asymmetry and type-I superconductivity in neutron star matter. Phys. Rev. C 2005, 72, 055801. [CrossRef]

35. Lalazissis, G.A.; Nikšić, T.; Vretenar, D.; Ring, P. New relativistic mean-field interaction with density-dependent meson-nucleon couplings. Phys. Rev. C 2005, 71, 024312. [CrossRef]

36. Ducoin, C.; Margueron, J.; Providência, C.; Vidaña, I. Core-crust transition in neutron stars: Predictivity of density developments. Phys. Rev. C 2011, 83, 045810. [CrossRef]

37. Wambach, J.; Ainsworth, T.L.; Pines, D. Quasiparticle interactions in neutron matter for applications in neutron stars. Nucl. Phys. A 1993, 555, 128-150. [CrossRef]

38. Baldo, M.; Elgarøy, Ø.; Engvik, L.; Hjorth-Jensen, M.; Schulze, H.J. ${ }^{3} \mathrm{P}_{2}-{ }^{3} \mathrm{~F}_{2}$ pairing in neutron matter with modern nucleon-nucleon potentials. Phys. Rev. C 1998, 58, 1921-1928. [CrossRef]

39. Baldo, M.; Cugnon, J.; Lejeune, A.; Lombardo, U. Proton and neutron superfluidity in neutron star matter. Nucl. Phys. A 1992, 536, 349-365. [CrossRef]

40. Sinha, M.; Sedrakian, A. Magnetar superconductivity versus magnetism: Neutrino cooling processes. Phys. Rev. C 2015, 91, 035805. [CrossRef]

41. Landau, L.D.; Lifshitz, E.M. Statistical Physics. Pt.2; Pergamon Press: Oxford, UK, 1980.

42. Lattimer, J.M.; Prakash, M.; Pethick, C.J.; Haensel, P. Direct URCA process in neutron stars. Phys. Rev. Lett. 1991, 66, 2701-2704. [CrossRef] [PubMed]

43. Pethick, C.J. Cooling of neutron stars. Rev. Mod. Phys. 1992, 64, 1133-1140. [CrossRef]

44. Prakash, M. Rapid cooling of neutron stars. Phys. Rep. 1994, 242, 297-312. [CrossRef] 
45. Bandyopadhyay, D.; Chakrabarty, S.; Dey, P.; Pal, S. Rapid cooling of magnetized neutron stars. Phys. Rev. D 1998, 58, 121301. [CrossRef]

46. Leinson, L.B.; Pérez, A. Direct URCA process in neutron stars with strong magnetic fields. J. High Energy Phys. 1998, 9, 20. [CrossRef]

47. Baiko, D.A.; Yakovlev, D.G. Direct URCA process in strong magnetic fields and neutron star cooling. Astron. Astrophys 1999, 342, 192-200.

48. Yakovlev, D.G.; Levenfish, K.P.; Shibanov, Y.A. Cooling of neutron stars and superfluidity in their cores. Phys. Uspekhi 1999, 42, 737-778. [CrossRef]

49. Sedrakian, A. The physics of dense hadronic matter and compact stars. Prog. Part. Nucl. Phys. 2007, 58, 168-246. [CrossRef]

50. Flowers, E.; Ruderman, M.; Sutherland, P. Neutrino pair emission from finite-temperature neutron superfluid and the cooling of young neutron stars. Asstrophys. Jour. 1976, 205, 541-544. [CrossRef]

51. Kaminker, A.D.; Haensel, P.; Yakovlev, D.G. Neutrino emission due to proton pairing in neutron stars. Astron. Astrophys. 1999, 345, L14-L16.

52. Leinson, L.B.; Pérez, A. Vector current conservation and neutrino emission from singlet-paired baryons in neutron stars. Phys. Lett. B 2006, 638, 114-118. [CrossRef]

53. Sedrakian, A.; Müther, H.; Schuck, P. Vertex renormalization of weak interactions and Cooper-pair breaking in cooling compact stars. Phys. Rev. C 2007, 76, 055805. [CrossRef]

54. Sedrakian, A. Vertex renormalization of weak interactions in compact stars: Beyond leading order. Phys. Rev. C 2012, 86, 025803. [CrossRef]

55. Kolomeitsev, E.E.; Voskresensky, D.N. Neutrino emission due to Cooper-pair recombination in neutron stars reexamined. Phys. Rev. C 2008, 77, 065808. [CrossRef]

56. Kolomeitsev, E.E.; Voskresensky, D.N. Neutral weak currents in nucleon superfluid Fermi liquids: Larkin-Migdal and Leggett approaches. Phys. Rev. C 2010, 81, 065801. [CrossRef]

(C) 2018 by the author. Licensee MDPI, Basel, Switzerland. This article is an open access article distributed under the terms and conditions of the Creative Commons Attribution (CC BY) license (http:/ / creativecommons.org/licenses/by/4.0/). 